\title{
EL USO DE LOS ORGANIZADORES DINÁMICOS DEL CONOCIMIENTO EN INVESTIGACIÓN EDUCATIVA EN EL NIVEL SUPERIOR
}

\author{
José Luis Castillo Córdova ${ }^{1}$
}

\section{RESÚMEN}

Se buscó determinar la eficacia de los organizadores dinámicos del conocimiento en el desarrollo de capacidades cognitivas en el Nivel Universitario. Se dictaron tres talleres a doscientos cuatro participantes, en los cuales se evaluó el grado de aceptación y los niveles de aprendizaje, respecto a la técnica desarrollada.Fue de nivel cuasi experimental con un solo grupo con pre y post test. Cada taller consistió en el uso de dinámicas y juegos realizados en base al uso didáctico (kirigami) del papel con aplicación en el nivel universitario. Se tuvo el cuidado de tocar temas de interés para cada especialidad. Como objetivo, buscamos desarrollar alternativas metodólogicas para el uso de los organizadores dinámicos del conocimiento como material educativo y de capacitación para todas las áreas de desarrollo, con énfasis en el área de comunicación. Se manejó como hipótesis: "El uso de los organizadores dinámicos del conocimiento desarrolla significativamente las capacidades comunicativas de los estudiantes y profesionales del nivel superior". Como conclusión podemos afirmar al $95 \%$ de confianza que el uso de los organizadores dinámicos del conocimiento influye en el aprendizaje de los alumnos del nivel universitario, especialmente en las capacidades comunicativas.

\section{ABSTRACT}

Dinamic organizar of knowledge are a technique based on the kirigami and maquigami techniques. Consist in use of articulated shapes of paper. Is economic, atractive and easy made. Has shown be efective as educative material for university level of education. The concepts is valid for multimedia presentations too.

Key Words: Organizadores del conocimiento, Material Educativo, Mapas mentales, aprendizaje, kirigami, maquigami, papel, creatividad, presentaciones.

\section{INTRODUCCIÓN}

Estando en boga el uso de organizadores visuales del conocimiento (mapas mentales, mapas conceptuales, círculo concéntrico, etc.) se presenta una alternativa atractiva y económica: los organizadores "dinámicos del movimiento".

Los organizadores dinámicos del conocimiento son una técnica basada en el kirigami y maquigami. Consiste en el uso de láminas de cartulina articuladas. Es un material muy económico, atractivo y simple.

Kirigami es el arte de recortar el papel, dibujando con las tijeras. Maquigami es el uso sólo de las manos con el mismo fin. Son técnicas desarrolladas en la

Universidad Nacional del centro y aplicadas desde el año 1990 en los niveles de inicial, primaria y Secundaria.

El presente trabajo de investigación buscó determinar la eficacia de los organizadores dinámicos del conocimiento en el desarrollo de capacidades cognitivas. Se dictaron talleres, en los cuales se evaluó el grado de aceptación y los niveles de aprendizajes alcanzados en el trabajo con 204 alumnos de diversas facultades de la UNCP y de la UPLA.

La presente investigación es de nivel cuasi experimental con un solo grupo con pre y post test.

En la formación universitaria y en las capacitaciones en el ámbito laboral generalmente se concibe que es suficiente una exposición frontal para el logro de los aprendizajes.

Por ello nos planteamos la siguiente interrogante:

¿Qué estrategias alternativas pueden dar resultados en el desarrollo de las capacidades comunicativas en el nivel superior y profesional?

Esta investigación pretende responder e esa pregunta a través del desarrollo de estrategias educativas alternativas en base al kirigami, como son los organizadores dinámicos del conocimiento.

Como objetivo, se busca en esta investigación desarrollar alternativas metodólogicas para el uso de los organizadores dinámicos como material educativo y de capacitación para todas las áreas de desarrollo, con énfasis en el área de comunicación, así como aplicarlos al desarrollo de optimas relaciones humanas y desarrollo personal. Todo esto dotando al alumno de nivel universitario de estrategias creativas que le permitan desarrollar capacidades comunicativas para afrontar con éxito los problemas de la vida cotidiana.

\section{Se maneja como hipótesis:}

"El uso de los organizadores dinámicos del conocimiento desarrolla significativamente las capacidades comunicativas de los estudiantes y profesionales del nivel superior"

Y tiene como variable independiente al Uso de los organizadores dinámicos del conocimiento, y como variable dependiente al Desarrollo de capacidades comunicativas.

Como conclusión podemos afirmar al $95 \%$ de confianza que el uso de los organizadores dinámicos del conocimiento influye en el aprendizaje de los alumnos del nivel universitario, especialmente en las capacidades comunicativas.

\footnotetext{
${ }^{1}$ Docente de la Escuela de Directores de IPAE. E: mail: kirigami@hotmail.com Telf. 98815600. WEB: http://mx.geocities.com/

kirigami1/. Universidad Nacional del Centro del Perú. Facultad de Arquitectura, Departamento de Teoria y Humanidades. Huancayo. 2001.
} 
Son muy gratificantes los logros alcanzados, pues nos motivan a seguir experimentando con nuevos métodos $y$ técnicas de enseñanza.

\section{MATERIAL Y MÉTODOS}

\section{TIPO DE INVESTIGACION}

La presente investigación será de nivel cuasi experimental con un solo grupo (Heterogéneo) con pre y post test.

\section{POBLACIÓN Y MUESTRA}

\section{POBLACIÓN}

Alumnos del nivel universitario de la ciudad de Huancayo.

\section{MUESTRA}

204 Alumnos de las especialidades de Ciencias de la Comunicación (UNCP), Enfermería (UNCP), Educación (UPLA) y contabilidad (UPLA).

\section{MÉTODO: Cuasi Experimental}

\section{DISEÑO: $\mathrm{O}_{1} \mathrm{XO}_{2}$}

Donde $X=$ Aplicación de los organizadores dinámicos del conocimiento.

\section{RESULTADOS}

El uso de los organizadores dinámicos del conocimiento es percibido como importante para el aprendizaje por los alumnos del nivel universitario, al $95 \%$ de confianza.

Estos resultados se dan independientemente de la carrera que cursen. Hay coherencia de los resultados en todas las especialidades estudiadas.

Los resultados del pre test demuestran que el estudiante de superior no valora, por lo general los materiales educativos simples, como el papel. Es posible que lo considere útil sólo para el trabajo en el nivel inicial y Primario. Pero Luego del taller realizado, su percepción del papel como material educativo dio un vuelco radical (Ver Gráficos 2 y 3).

\section{DISCUSIÓN}

Los resultados obtenidos en el pre test $y$ en el post test luego de aplicada la variable organizadores dinámicos del conocimiento nos indican que al $95 \%$ de confianza, son percibidos como importantes para el aprendizaje por los alumnos del nivel universitario.

\begin{tabular}{|l|c|c|c|c|}
\cline { 2 - 5 } \multicolumn{1}{c|}{} & $\mathrm{N}$ & Mean & $\begin{array}{c}\text { Std. } \\
\text { Deviation }\end{array}$ & $\begin{array}{c}\text { Std. Error } \\
\text { Mean }\end{array}$ \\
\hline Habilidad comunicativa PRE & 204 & 3,7696 & 1,3357 & $9,352 \mathrm{E}-02$ \\
\hline Habilidad comunicativa POST & 174 & 4,4770 & 0,9954 & $7,546 \mathrm{E}-02$ \\
\hline
\end{tabular}

Cuadro 1: One-Sample Statistics

$95 \%$

Confidence

Interval of the

Difference

\begin{tabular}{|l|c|c|c|c|c|c|}
\cline { 2 - 7 } \multicolumn{1}{c|}{} & $\mathrm{t}$ & $\mathrm{df}$ & $\begin{array}{c}\text { Sig. } \\
(2-\text {-tailed })\end{array}$ & $\begin{array}{c}\text { Mean } \\
\text { Difference }\end{array}$ & Lower & Upper \\
\hline Habilidad comunicativa PRE & 40,309 & 203 & 0,00 & 37,696 & 35,852 & 39,540 \\
\hline Habilidad comunicativa POST & 59,329 & 173 & 0 & 44,770 & 43,281 & 46,260 \\
\hline
\end{tabular}

Cuadro 2: One-Sample Test

\begin{tabular}{|l|c|c|c|c|}
\hline & $\mathrm{N}$ & Mean & Std. Deviation & Std. Error Mean \\
\hline Pre Test & 8 & 3,125000 & 0,561306 & 0,198452 \\
\hline Post Test & 8 & 4,050288 & 0,313974 & 0,111006 \\
\hline
\end{tabular}

Cuadro 3: Comparación entre el pre test y post test globales considerando la media de las 8 medias obtenidas. One-Sample Statistics

\begin{tabular}{|l|c|c|c|c|c|c|}
\cline { 3 - 7 } \multicolumn{1}{c}{} & \multicolumn{4}{c}{} & \multicolumn{2}{|c|}{$\begin{array}{c}95 \% \text { Confidence Interval } \\
\text { of the Difference }\end{array}$} \\
\cline { 2 - 7 } \multicolumn{1}{c|}{$\mathrm{t}$} & $\mathrm{df}$ & $\begin{array}{c}\text { Sig. } \\
(2-\text {-tailed })\end{array}$ & $\begin{array}{c}\text { Mean } \\
\text { Difference }\end{array}$ & Lower & Upper \\
\hline Pre Test & 15,747 & 7 & 0,00 & $3,125,000$ & $2,655,736$ & $3,594,264$ \\
\hline Post Test & 36,487 & 7 & 0,00 & $4,050,288$ & $3,787,799$ & $4,312,776$ \\
\hline
\end{tabular}

Cuadro 4: One-Sample Test Test Value $=0$

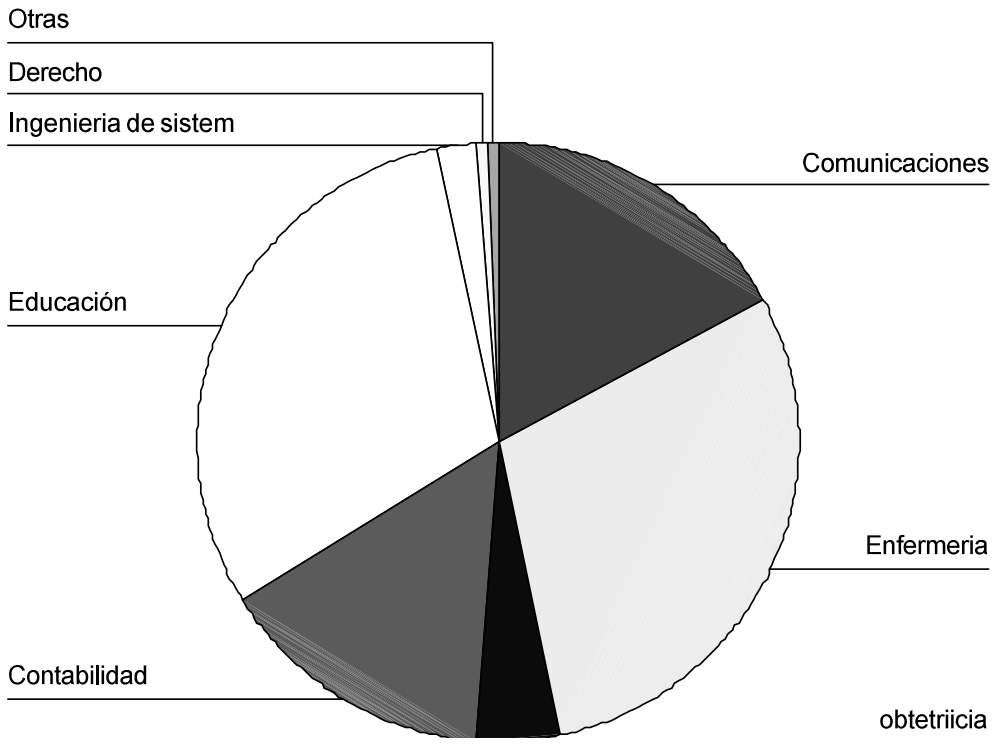

Gráfico 1: distribución por especialidades de la muestra 


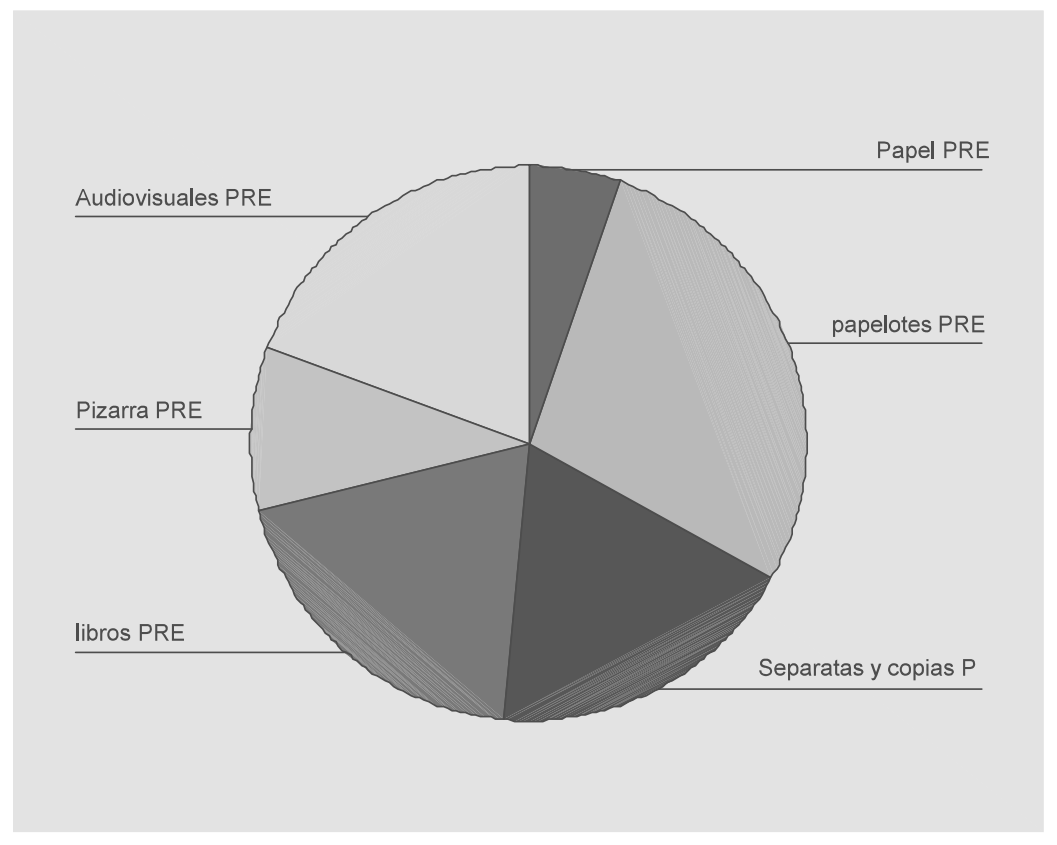

Grafico 2: Materiales sugeridos Pre Test.

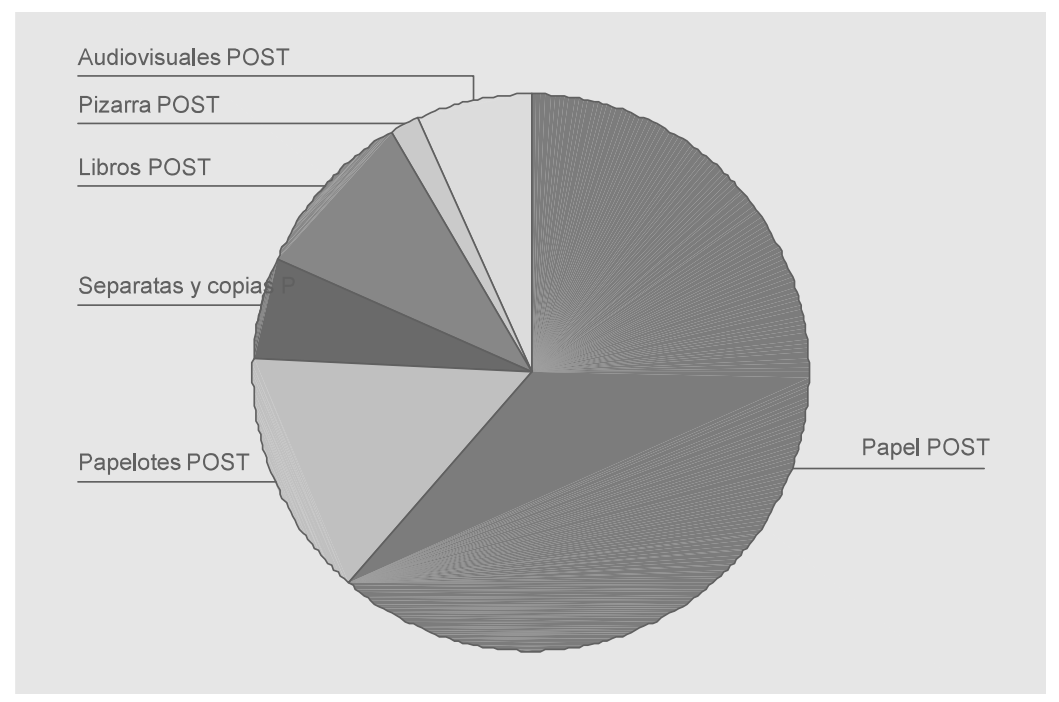

Grafico 3: Materiales sugeridos Post test. Organizadores (Papel)

\section{AGRADECIMIENTOS}

A los alumnos de la Universidad Nacional del Centro del Perú por el entusiasmo demostrado durante la realización de los talleres.

En las fotografías vemos algunos modelos de organizadores dinámicos desarrollados.

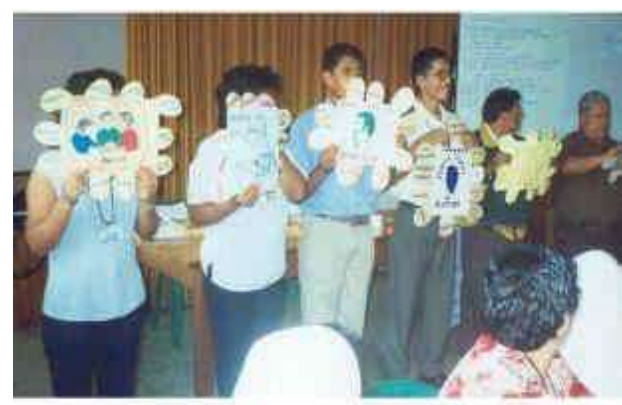

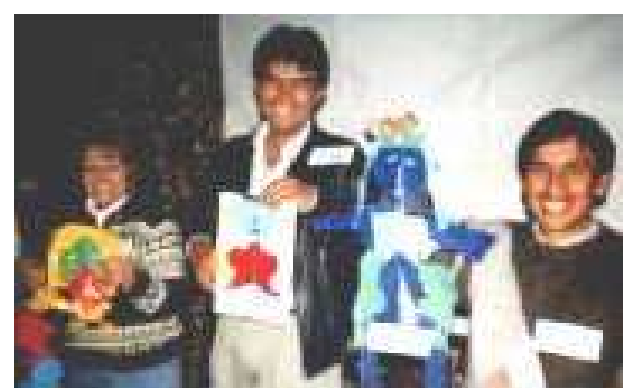

\section{REFERENCIAS BIBLIOGRÁFICAS}

1. Alarcón Ureta G, Amtialón Arana D. "Materiales didácticos de física construídos en base a papel para mejorar el aprendizaje de la dinámica en las alumnas del quinto grado de educación secundaria del Colegio Estatal "Nuestra Señora del Cocharcas". UNCP. 2000.

2. Castillo Córdova JL. "KIRIGAMI Y MAQUIGAMI: La Magia del Papel". Quinta Edición. Huancayo. Maquihuasi 2001.

3. Ccanto Condori DL, Espinoza Roque B. "Los Juegos dramáticos con papel y sus efectos en la autoestima de las niñas del quinto grado de educación primaria del Centro Educativo "Sagrado Corazón de Jesús - Huancayo".

UNCP, 2000.

4. Damián Munguía L, Matos Manrique E. "Técnica de figuras recortables de papel en el desarrollo de habilidades básicas para la lecto escritura de los alumnos del J.N.E. N498 La Victorial - El Tambo". Huancayo, UNCP 2000 